\section{Multifunctional microbubbles for image-guided antivascular endothelial growth factor therapy}

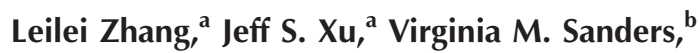 \\ Alan D. Letson, ${ }^{c}$ Cynthia J. Roberts, ${ }^{a, c}$ and \\ Ronald X. X $\mathbf{u}^{\mathrm{a}, \mathrm{c}}$, \\ ${ }^{a}$ The Ohio State University, Department of Biomedical \\ Engineering, \\ ${ }^{\mathrm{b}}$ Department of Molecular Virology, Immunology \& Medical \\ Genetics, \\ ${ }^{\mathrm{c}}$ Department of Ophthalmology, Columbus, Ohio 43210
}

\begin{abstract}
We synthesize multifunctional microbubbles (MBs) for targeted delivery of antivascular endothelial growth factor (antiVEGF) therapy with multimodal imaging guidance. Poly-lactic-Co-glycolic acid (PLGA) MBs encapsulating Texas Red dye are fabricated by a modified double-emulsion process. Simultaneous ultrasound and fluorescence imaging are achieved using Texas Red encapsulated MBs. The MBs are conjugated with Avastin, an antiVEGF antibody for treating neovascular age-related macular degeneration (AMD). The conjugation efficiency is characterized by enzyme-linked immunosorbent assay (ELISA). The efficiency for targeted binding of Avastinconjugated $\mathrm{MBs}$ is characterized by microscopic imaging. Our work demonstrates the technical potential of using multifunctional MBs for targeted delivery of antiVEGF therapy in the treatment of exudative AMD. $\odot 2010$ Society of Photo-Optical Instrumentation Engineers. [DOI: 10.1117/1.3457669]
\end{abstract}

Keywords: microbubble; age-related macular degeneration; fluorescence; ultrasound; multimodal imaging; Avastin; bevacizumab; drug delivery.

Paper 10234LR received May 1, 2010; revised manuscript received May 27, 2010; accepted for publication May 31, 2010; published online Jul. 1, 2010.

Age-related macular degeneration (AMD) is the leading cause of vision loss and blindness in people over age 65 in industrialized nations. ${ }^{1-3}$ Exudative AMD, which is characterized by choroidal neovascularization $(\mathrm{CNV})$ and retina pigment epithelium (RPE) detachments, causes 80 to $90 \%$ of cases of severe vision loss relative to AMD. ${ }^{4}$ Since the vascular endothelial growth factor (VEGF) plays an important role in the development of neovascular AMD, various antiVEGF drugs such as Lucentis (ranibizumab) and Macugen (pegaptanib) have been approved by the Federal Drug Administration (FDA) for the clinical treatment of AMD. ${ }^{5}$ Although Avastin (Genetech, San Francisco, California) (bevacizumab) has not been approved by FDA for use in the eye, it is used widely off-label by ophthalmologists. ${ }^{6}$ It is now recognized as a standard of care and is covered by third-party insurance, including Medicare (the United States Government) and most

Address all correspondence to: Ronald X. Xu, Tel: 614-688-3635; Fax: 614-2927301; E-Mail: xu.202@osu.edu other third-party payers. Avastin is typically administrated by intravitreous injection, a widely accepted and relatively safe procedure when performed following the protocol. ${ }^{7}$ However, a single intravitreous injection cannot sustain a high level of intraocular drug concentration for a long time. Therefore, frequent injection is necessary. Frequent injections may not only increase the risk of complications, but also introduce logistical and cost concerns as well as be an inconvenience for patients, their families, and care-delivery systems. In addition to dose frequency, dose level may also play an important role in antiVEGF therapy of AMD, which is currently being evaluated by Genentech in an ongoing clinical trial. However, appropriate dosage control is difficult to achieve due to the lack of quantitative imaging tools.

Recent advances in micro- and nanodrug-delivery devices have opened a new avenue for targeted pharmacotherapeutics of neovascular AMD. ${ }^{8}$ Many drug-delivery devices have been synthesized using biocompatible and biodegradable materials, such as liposome, albumin, polylactide (PLA), and polylactic-co-glycolic acid (PLGA). PLGA has been approved by the FDA for implantation applications, and is now widely investigated as a biodegradable carrier for controlled drug release. ${ }^{9}$ We have previously encapsulated absorption and fluorescence dyes in PLGA microbubbles (MBs) and nanobubbles (NBs) for multimodal imaging using different modalities such as fluorescence (FL), ultrasound (US), and photoacoustic tomography (PAT). ${ }^{10,11}$ We have also modified and conjugated PLGA MBs and NBs for cancer targeting and imaging. ${ }^{12}$ Similar fabrication and conjugation techniques can be applied to synthesize drug-loaded microdevices for imageguided antiVEGF therapy.

This work reports our recent effort on synthesizing multifunctional PLGA MBs for targeted pharmacotherapeutics of neovascular AMD. Carboxylic acid terminated PLGA (50:50, RG 502H 12,000 Da MW, Boehringer Ingelheim, Germany) MBs encapsulating Texas Red (AnaSpec Incorporated, San Jose, California) were fabricated following a modified doubleemulsion process. ${ }^{12}$ Briefly, 0.5-mL solution with $40-\mu \mathrm{M}$ Texas Red and 4\% w/v polyvinyl alcohol (PVA, Fisher Scientific, Pittsburgh, Pennsylvania) was added to 5-mL methylene chloride $\left(\mathrm{CH}_{2} \mathrm{Cl}_{2}\right.$, Fisher Scientific) dissolving 125-mg carboxylic acid terminated PLGA for the first emulsion. Then, the suspension was added to $50 \mathrm{~mL}$ of $4 \% \mathrm{w} / \mathrm{v}$ PVA for the second emulsion. After $\mathrm{CH}_{2} \mathrm{Cl}_{2}$ was extracted by adding $100 \mathrm{~mL}$ of $5 \% \mathrm{v} / \mathrm{v}$ isopropanol aqueous solution and stirring for three hours, the resultant suspension of the hardened MBs was centrifuged (Eppendorf Centrifuge 5810R, Hamburg, Germany). The MB precipitates at the centrifuge speed between 1000 and $2000 \mathrm{rpm}$ were collected, freeze dried, and stored at $0{ }^{\circ} \mathrm{C}$ for further use.

Figure 1 shows the scanning electron microscope (SEM) image of the fabricated MBs. The size of these MBs was $1389 \pm 276 \mathrm{~nm}$ and the Zeta potential was $-7.83 \pm 0.82 \mathrm{mV}$, as characterized by a Dynamic Laser Scattering System (BI200SM, Brookhaven Instruments Corporation, New York). MBs with this size range were used to facilitate effective intraocular drug delivery with large drug loading efficiency, strong ultrasound contrast, and minimal vision blockage. MB suspensions at concentrations of $5,2.5$, and $1.25 \mathrm{mg} / \mathrm{mL}$

1083-3668/2010/15(3)/030515/3/\$25.00 @ 2010 SPIE 


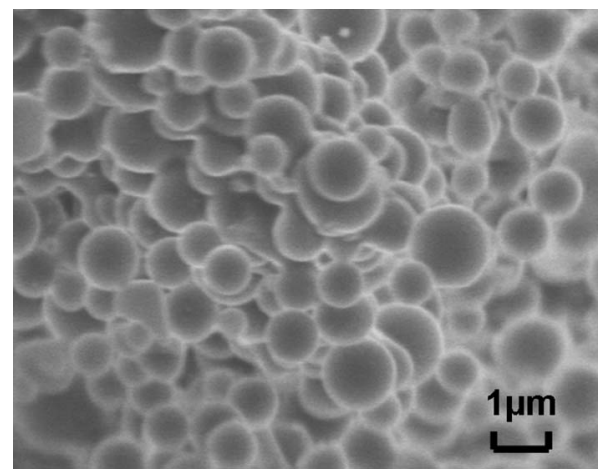

Fig. 1 A typical SEM image of Texas Red encapsulated MBs.

were filled in three centrifuge tubes respectively for FL and US imaging. An additional centrifuge tube filled with water was used as the control. FL images were acquired using a Maestro EX Fluorescence imaging system (CRi, Woburn, Massachusetts) at the excitation wavelength of $590 \mathrm{~nm}$ and an emission wavelength of $610 \mathrm{~nm}$, as shown in Fig. 2(a). Immediately after FL imaging, US images were acquired by a Vevo 2100 US system (VisualSonics Incorporated, Toronto, Canada) with a 20-MHz transducer, as shown in Fig. 2(b). FL and US image intensities were estimated at different MB concentrations, as plotted in Fig. 3. Linear correlations were observed between the FL intensity and the MB concentration $\left(R^{2}=0.960\right)$ and between the US intensity and the MB concentration $\left(R^{2}=0.991\right)$, indicating the technical potential of using FL and US imaging for doximetric assessment and control.

The carboxylic acid terminated PLGA MBs were surface modified and Avastin conjugated for VEGF targeting and drug delivery. Briefly, MBs were dispersed in a 2-(N-morpholino) ethanesulfonic acid (MES) buffer (0.1 M, pH=5.6) at concentrations of $5,2.5$, and $1.25 \mathrm{mg} / \mathrm{mL}$, respectively. Then, 1-ethyl-3(3-dimethylaminopropyl) carbodiimide hydrochloride (EDC) was added into MBs suspensions to a final concentration of $2 \mathrm{mM}$. Immediately after that, N-hydroxy succinimide (NHS) was added to a final concentration of $5 \mathrm{mM}$. The suspensions were stirred slightly for $15 \mathrm{~min}$ and washed by MES buffer three times. The precipitates were then col-

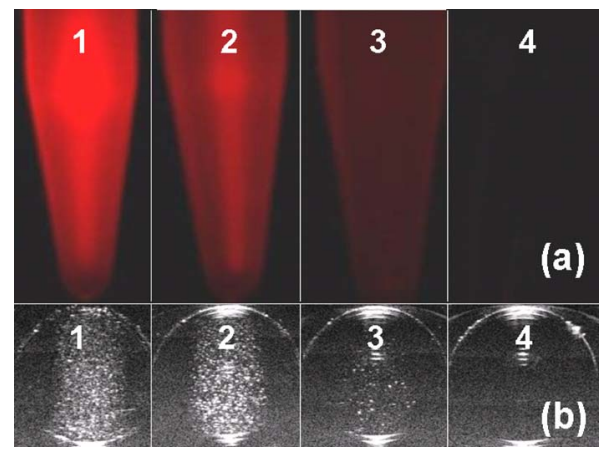

Fig. $2 \mathrm{FL}$ and US images of Texas Red encapsulated MBs stored in different centrifuge tubes. The $\mathrm{MB}$ concentrations from left to right are: $1.5 \mathrm{mg} / \mathrm{mL}, 2.2 .5 \mathrm{mg} / \mathrm{mL}, 3.1 .25 \mathrm{mg} / \mathrm{mL}$, and $4.0 \mathrm{mg} / \mathrm{mL}$. (a) FL images of the centrifuge tubes (front view). (b) The corresponding US images (cross sectional view).

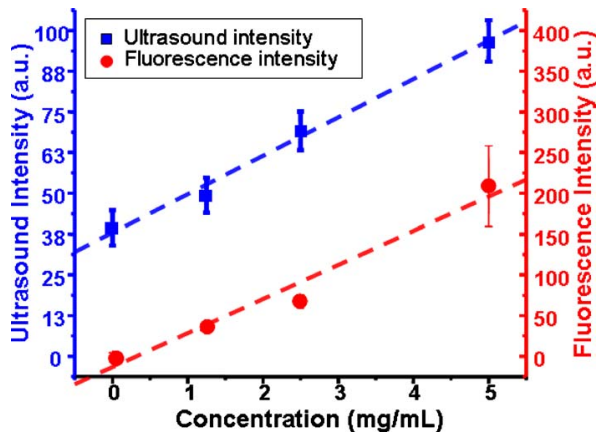

Fig. 3 FL intensities (red dots) and US intensities (blue rectangles) of Texas Red encapsulated MBs at the following MB concentration levels: $0,1.25,2.5$, and $5 \mathrm{mg} / \mathrm{mL}$. (Color online only.)

lected and redispersed in phosphate buffered saline (1 $\times$ PBS, $\mathrm{PH}=7.4)$. To conjugate Avastin with NHS-ester active MBs, Avastin (Genetech, San Francisco, California) was added into the $\mathrm{MB}$ suspension to a final concentration of $167 \mu \mathrm{g} / \mathrm{mL}$. After that, the suspension was stirred slightly at room temperature for $2 \mathrm{~h}$ followed by centrifugation at $5000 \mathrm{rpm}$ for $5 \mathrm{~min}$. The supernatant was collected for enzyme-linked immunosorbent assay (ELISA) assessment of the conjugation efficiency, while the precipitate was collected and redispersed in $1 \times$ PBS for further binding tests.

The Avastin conjugation efficiency was determined by an indirect ELISA method, where the concentration of supernatant Avastin without reactivation with MBs was measured. The conjugation efficiency was derived by subtracting the supernatant Avastin concentration from the total concentration. Briefly, the 96-well ELISA plate (Fishers) was first coated with $15-\mu \mathrm{g} / \mathrm{mL}$ monoclonal antihuman IgG1 (Fab specific) antibody (Sigma, Saint Louis, Missouri) in 50-mM carbonate buffer $(\mathrm{pH}=9.6)$ at room temperature overnight, and then blocked by $1 \%$ bovine serum albumin (BSA) for $1 \mathrm{~h}$. The bound Avastin was detected by using an antihuman IgG antibody (Fc specific) with alkaline phosphatase (Sigma) on a SpectraMax Plus microplate reader (Molecular Devices, Sunnyvale, California). Figure 4 shows the standard ELISA curve fitted by 4-P logistic regression $\left(R^{2}=0.996\right)$. The Avastin conjugation efficiencies at MB concentration levels of $1.25,2.5$, and $5 \mathrm{mg} / \mathrm{mL}$ are $70.22,47.20$, and $57.35 \%$, respectively, as shown in Fig. 4.

The ELISA plate was also used to test the targeted binding efficiency of Avastin conjugated MBs. The monoclonal antihuman IgG1 (Fab specific) antibody was first incubated in the wells of the ELISA plate overnight and then blocked by $1 \%$ BSA. Instead of using the supernatant, the redispersed MBs after conjugation were incubated in the wells at concentration levels of $5,2.5$, and $1.25 \mathrm{mg} / \mathrm{mL}$, respectively. Meanwhile, the control MBs without Avastin conjugation were also incubated at the same concentration levels in different wells of the same plate. After an overnight incubation at room temperature, the plate was washed six times with $0.05 \%$ Tween-20 in $1 \times$ PBS and then imaged by an inverted microscope. MB distributions for both the samples and the controls were estimated at three concentration levels. The density of distributed MBs was then averaged in six randomly selected regions of interests for each test case. As shown in Fig. 5, the Avastin conjugated MBs (sample) have greater targeted binding effi- 


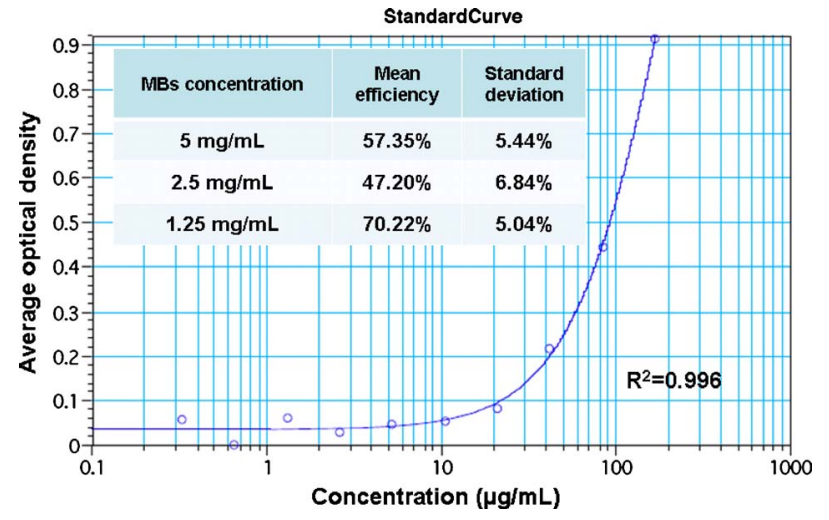

Fig. 4 The standard ELISA curve (the blue line) and the conjugation efficiencies at different MB concentration levels (the embedded table). The standard ELISA curve was fitted by 4-P logistic regression with $R^{2}=0.996$. A typical plateau at the high concentrations cannot be observed due to the limited Avastin supply available in this experiment. The table shows the averaged conjugation efficiency and deviation at different MB concentrations. (Color online only.)

ciency than bare MBs (control). The targeted binding efficiency for Avastin conjugated MBs is significantly higher than that for bare MBs at the MB concentration level of $5 \mathrm{mg} / \mathrm{mL}$.

In summary, we synthesize a multifunctional MB agent that can potentially be used for image-guided antiVEGF therapy in exudative AMD. Our next step is to encapsulate biodegradable dyes such as indocyanine green (ICG) in MBs for in vitro and in vivo validation of this image-guided drug delivery schema. Since microbubbles have strong scattering contrast for optical coherence tomography $(\mathrm{OCT}),{ }^{13}$ it is also possible to use OCT for image-guided intravitreous delivery of drug-loaded MBs. In addition to drug-loaded MBs, we also

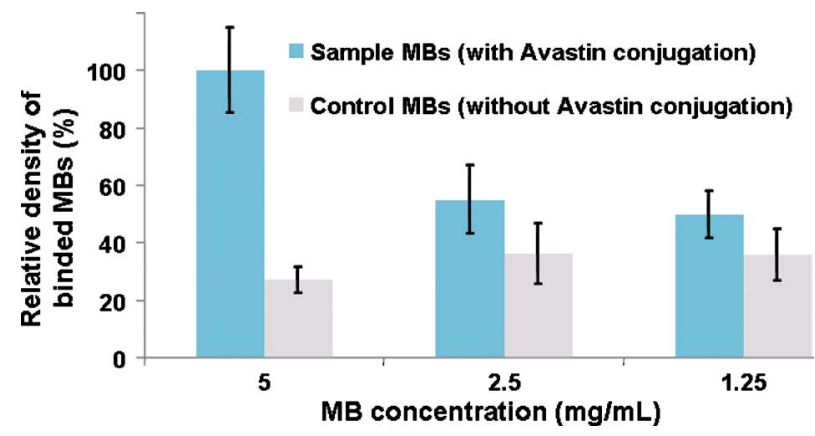

Fig. 5 Comparison of relative MB densities after the binding tests for the sample MBs (with Avastin conjugation) and the control MBs (without Avastin conjugation) at different concentration levels. The density of Avastin conjugated MBs at the concentration of $5 \mathrm{mg} / \mathrm{mL}$ was normalized as $100 \%$. fabricate MBs that can be activated by an external energy source. ${ }^{14}$ The MB targeting mechanism described in this work may facilitate the sustained release of antiVEGF agents by sequential ultrasound sessions in the later time.

\section{Acknowledgments}

The authors are grateful for the fluorescence imaging assistance from Michael Tweedle and Michelle Carlton in the Department of Radiology, for the data processing assistance by Jiwei Huang in the Biophysics Graduate Program, and for the technical support by Scot Erbe in the Department of Molecular Virology, Immunology and Medical Genetics.

\section{References}

1. R. Klein, B. E. Klein, and K. L. Linton, "Prevalence of age-related maculopathy. The Beaver Dam Eye Study," Ophthalmology 99(6), 933-943 (1992).

2. C. A. Augood, J. R. Vingerling, P. T. de Jong, U. Chakravarthy, J. Seland, G. Soubrane, L. Tomazzoli, F. Topouzis, G. Bentham, M. Rahu, J. Vioque, I. S. Young, and A. E. Fletcher, "Prevalence of age-related maculopathy in older Europeans: the European Eye Study (EUREYE)," Arch. Ophthalmol. (Chicago) 124(4), 529-535 (2006).

3. P. Mitchell, W. Smith, K. Attebo, and J. J. Wang, "Prevalence of age-related maculopathy in Australia. The Blue Mountains Eye Study," Ophthalmology 102(10), 1450-1460 (1995).

4. M. Votruba and Z. Gregor, "Neovascular age-related macular degeneration: present and future treatment options," Eye 15(Pt 3), 424-429 (2001).

5. J. P. Hubschman, S. Reddy, and S. D. Schwartz, "Age-related macular degeneration: current treatment," Clin. Ophthalmol. 3, 155-166 (2009).

6. R. Steinbrook, "The price of sight-ranibizumab, bevacizumab, and the treatment of macular degeneration," N. Engl. J. Med. 355(14), 1409-1412 (2006).

7. J. P. Hubschman, S. Reddy, and S. D. Schwartz, "Age-related macular degeneration: experimental and emerging treatments," Clin. Ophthalmol. 3, 167-174 (2009).

8. A. A. Moshfeghi and G. A. Peyman, "Micro- and nanoparticulates," Adv. Drug Delivery Rev. 57(14), 2047-2052 (2005).

9. R. A. Jain, "The manufacturing techniques of various drug loaded biodegradable poly(lactide-co-glycolide) (PLGA) devices," Biomaterials 21(23), 2475-2490 (2000).

10. R. Xu, J. Huang, J. Xu, D. Sun, G. Hinkle, J. Edward, W. Martin, and S. Povoski, "Fabrication of indocyanine green encapsulated biodegradable microbubbles for structural and functional imaging of cancer," J. Biomed. Opt. 14(3), 034020 (2009).

11. C. Kim, R. Qin, J. S. Xu, L. Wang, and R. X. Xu, "Ultrasound and photoacoustic dual-modal imaging of thick biological tissue with microbubble enhancement," J. Biomed. Opt. 15(1), 010510 (2010).

12. J. S. Xu, J. Huang, R. Qin, G. H. Hinkle, S. P. Povoski, E. W. Martin, and R. X. Xu, "Synthesizing and binding dual-mode poly (lactic-coglycolic acid) (PLGA) nanobubbles for cancer targeting and imaging," Biomaterials 31(7), 1716-1722 (2010).

13. J. K. Barton, J. B. Hoying, and C. J. Sullivan, "Use of microbubbles as an optical coherence tomography contrast agent," Acad. Radiol. 9(suppl 1), S52-55 (2002).

14. J. Huang, J. S. Xu, and R. X. Xu, "Heat-sensitive microbubbles for intraoperative assessment of cancer ablation margins," Biomaterials 31(6), 1278-1286 (2010). 